\title{
PARAMETER ESTIMATION FOR A SHIP'S ROLL RESPONSE MODEL IN SHALLOW WATER USING AN INTELLIGENT MACHINE LEARNING METHOD
}

\author{
Changyuan Chen and Manases Tello Ruiz, Ghent University, Belgium \\ Guillaume Delefortrie, Flanders Hydraulics Research and Ghent University, Belgium \\ Tianlong Mei, Shanghai Jiao Tong University, China \\ Evert Lataire and Marc Vantorre, Ghent University, Belgium
}

\section{SUMMARY}

In order to accurately identify the ship's roll model parameters in shallow water, and solve the problems of difficult estimating nonlinear damping coefficients by traditional methods, a novel Nonlinear Least Squares - Support Vector Machine (NLS-SVM) is introduced. To illustrate the validity and applicability of the proposed method, simulation data and decay tests data are combined and utilized to estimate unknown parameters and predict the roll motions. Firstly, simulation data is applied in the NLS-SVM model to obtain estimated damping parameters, compared with pre-defined parameters to verify the validity of the proposed method. Subsequently, decay tests data are used in identifying unknown parameters by utilizing traditional models and the new NLS-SVM model, the results illustrate that the intelligent method can improve the accuracy of parametric estimation, and overcome the conventional algorithms' weakness of difficult identification of the nonlinear damping parameter in the roll model. Finally, to show the wide applicability of the proposed model in shallow water, experimental data from various speeds and Under Keel Clearances (UKCs) are applied to identify the damping coefficients. Results reveal the potential of using the NLS-SVM for the problem of the roll motion in shallow water, and the effectiveness and accuracy are verified as well.

Keywords: roll model; shallow water; NLS-SVM; damping parameters; parameter identification

\section{INTRODUCTION}

Roll motion is one of the most critical responses the ship experiences in her lifespan. An accurate prediction of its development in real scenarios is then deemed necessary so to understand better the ship behavior and to avoid any hazardous conditions (Jiang et al., 2016; Yin et al., 2018). For this purpose, the identification of the roll viscous damping's contribution is of critical interest (Sun and Sun, 2013; Jiang et al., 2017). The most common approach to obtain this component is by means of free roll decay tests and fitting the measured response by conventional methods such as Least Squares (LS) approach. This method, however, when applied in shallow water has some difficulties to provide a clear distinction of the viscous parameters, especially in the nonlinear term. One may argue that other alternatives, available nowadays, can provide better results (especially for the nonlinear damping term).

The paper aims to investigate novel intelligent identification approaches which can be compared and tested against conventional methods. In the present work, the NLS-SVM algorithm will be investigated. Compared with other intelligent approaches, using large samples of data to estimate unknown parameters, NLS-SVM only depends on limited support vectors based on small samples. Besides, the structure risk minimization theory instead of empirical risk minimization - is adopted by NLS-SVM to solve optimization problems. A global optimization result is obtained, and local optimization issues are avoided. Moreover, high accuracy, time saving and wide applicability performances of NLS-SVM are especially suitable for the identification of damping parameters in shallow water.
To verify the effectiveness, accuracy and applicability of the NLS-SVM parametric estimation model in shallow water, free roll decay tests for a scale model of an Ultra Large Container Vessel (ULCV) at different forward speeds and different UKCs were carried out. Then, simulation and experimental data are used to identify and predict the ship's roll motions. Comparisons between predicted data and original data illustrate the potential of employing the proposed method for the problem of roll motion in shallow water.

\section{SHIP ROLL HYDRODYNAMIC MODEL}

In a sense, an accurate definition and prediction of damping parameters (especially nonlinear damping coefficients) in the ship roll model is a very necessary task (Hou and Zou, 2016; Hou et al., 2018). On the basis of the rigid body theory (Hou and Zou, 2015), the 1DOF ship roll motion model can be written as (Xing and McCue, 2010):

$$
\left(I_{x x}+A_{44}^{\infty}\right) \ddot{\phi}+B(\dot{\phi})+C_{44} \phi=0
$$

where $I_{x x}$ is the mass moment of inertia, $A_{44}^{\infty}$ is the added mass moment of inertia (at infinite frequency), $B$ is the moment due to the damping phenomena, $C_{44}$ is the roll restoring coefficient, and $\phi$ is the roll angle. The single dotted and double dotted variables represent the first and second order derivatives.

The total damping coefficients are divided into a linear $\left(b_{\dot{\phi}}\right)$, a nonlinear $\left(b_{\dot{\phi}|\dot{\phi}|}\right)$ (Ikeda et al., 1977; Himeno, 1981), and a potential contribution components in the following form:

$$
\begin{aligned}
& B(\dot{\phi})=b_{\dot{\phi}} \dot{\phi}+b_{\dot{\phi}|\dot{\phi}|} \dot{\phi}|\dot{\phi}|+\int_{-\infty}^{+\infty} h_{44}(t- \\
& \tau) \dot{\phi}(\tau) d \tau
\end{aligned}
$$


where $\dot{\phi}$ is the roll rate and $h_{44}$ is the impulse response function (IRF). Substituting $B(\dot{\phi})$ into Eq. (1), the final model in the time domain is expressed as follows:

$$
\begin{aligned}
& \left(I_{x x}+A_{44}^{\infty}\right) \ddot{\phi}+b_{\dot{\phi}} \dot{\phi}+b_{\dot{\phi}|\dot{\phi}|} \dot{\phi}|\dot{\phi}|+ \\
& +\int_{-\infty}^{+\infty} h_{44}(t-\tau) \dot{\phi}(\tau) d \tau+C_{44} \phi=0
\end{aligned}
$$

\section{PARAMETERS ESTIMATION APPROACHE}

\subsection{PROBLEM STATEMENT}

Assuming that a parametric system in state-form is available in the form of:

$$
\frac{d X}{d t}=g(X, T, \theta)
$$

Where $X=\left[x_{1} ; x_{2} ; \ldots ; x_{i}\right]$ is the state variable, $\frac{d X}{d t}=$ $\left[\frac{d}{d t} x_{1}, \frac{d}{d t} x_{2}, \ldots, \frac{d}{d t} x_{i}\right]^{T}$ is the derivative of each state variable, $T=\left[t_{1}, t_{2}, \ldots, t_{i}\right]^{T}$ is the time variable, $\theta=$ $\left[\theta_{1}, \theta_{2}, \ldots, \theta_{i}\right]^{T}$ is an unknown set of parameters (Mehrkanoon et al., 2012).

For the parametric system, the main goal is to identify the unknown parameters $\theta$ from observed data $Y=$ $\left[y_{1}, y_{2}, \ldots, y_{i}\right]^{T}$ at time variable $T=\left[t_{1}, t_{2}, \ldots, t_{i}\right]^{T}$.

$$
e_{i}=Y\left(t_{i}\right)-X\left(t_{i}\right), i=1,2 \cdots n
$$

where $e_{i}=\left[e_{1}, e_{2}, \ldots, e_{i}\right]^{T}$ is the error between observed data $Y$ and outputs of the estimate state variable $X$. The final goal is shifted to get the set of unknown parameters by minimizing the error $e_{i}$.

\subsection{IDENTIFICATION PROCEDURE}

\section{Step 1 Obtain sample data}

Obtain training samples data $\left\{\left(t_{i}, y_{i}\right), i=1,2, \ldots, n\right\}$, where $t_{i}$ is the time series, and $y_{i}$ is the numerical simulation data or experimental data.

\section{Step 2 Approximate the state variable}

Estimate the state variable $\hat{X}=\left[\hat{x}_{1}, \hat{x}_{2}, \ldots, \hat{x}_{i}\right]^{T}$ based on simulation or experimental data $\left\{\left(t_{i}, y_{i}\right), i=1,2, \cdots n\right\}$. In the present study, NLS-SVM is adopted to approximate the state variable $\hat{X} . x_{k}$ or the $k$-th state variable can be obtained by an approximation function of the following form:

$$
\hat{x}_{k}(t)=w_{k}^{T} \varphi(t)+b_{k}
$$

where $t$ is the input data (time), $\hat{x}_{k}$ is the output data, $w_{k}$ is the weight value, $\varphi(\cdot)$ is the nonlinear function, which maps the input data $t$ to the Euclidean space, $b_{k}$ is the bias (David et al., 2013; Xu and Guedes Soares, 2016).

To solve the convex optimization issue according to the minimization of structure risk theory, construct and solve the following cost function:

$$
\min _{w, b, e} f(w, e)=\frac{1}{2} w^{T} w+\frac{1}{2} \gamma\left\|e_{i}\right\|_{2}^{2}
$$

Subject to

$$
y_{i}=w^{T} \varphi\left(t_{i}\right)+b+e_{i}
$$

where $i=1,2, \cdots, \mathrm{n}, \gamma$ is penalty factor, $e_{i}$ is the error.

A lagrangian function is introduced to solve the optimization problem as follows:

$$
\begin{aligned}
& \mathrm{L}(w, b, e, a)=\frac{1}{2} w^{T} w+\frac{1}{2} \gamma\left\|e_{i}\right\|_{2}^{2}- \\
& \sum_{i=1}^{n} a_{i}\left[w^{T} \varphi\left(t_{i}\right)+b+e_{i}-y_{i}\right]
\end{aligned}
$$

where the coefficients $a_{i}$ are the Lagrange multipliers. The derivative matrix is obtained by partially differentiating Eq. (9) with respect to $w, b, e, a$ :

$$
\left[\begin{array}{cc}
K+\gamma^{-1} I_{N} & 1_{N} \\
1_{N}^{T} & 0
\end{array}\right]\left[\begin{array}{l}
a^{k} \\
b^{k}
\end{array}\right]=\left[\begin{array}{c}
y^{k} \\
0
\end{array}\right]
$$

where $K\left(t_{i}, t_{j}\right)=\varphi\left(t_{i}\right) \varphi\left(t_{j}\right)$ is the kernel function, $I_{N}$ is the identity matrix, $1_{N}=[1 ; 1 ; \ldots ; 1], \quad \alpha^{k}=$ $\left[a_{1}^{k} ; a_{2}^{k} ; \ldots ; a_{n}^{k}\right]$.

The regression model is expressed as

$$
\hat{x}_{k}(t)=w_{k}^{T} \varphi(t)+b_{k}=\sum_{i=1}^{n} \alpha_{i}^{k} K\left(t_{i}, t\right)+b_{k}
$$

Step 3 Derivatives of the state variable approximation Approximate the derivatives of the state variable $\frac{d}{d t} \hat{X}=$ $\left[\frac{d}{d t} \hat{x}_{1}, \frac{d}{d t} \hat{x}_{2}, \ldots, \frac{d}{d t} \hat{x}_{n}\right]$ by differentiating the approximated model with time.

Differentiation of $\hat{x}_{k}(t)$ with time, yields:

$$
\begin{aligned}
& \frac{d}{d t} \hat{x}_{k}(t)=w_{k}^{T} \dot{\varphi}(t)= \\
& \sum_{i=1}^{n} \alpha_{i}^{k} \varphi\left(t_{i}\right)^{T} \dot{\varphi}(t)
\end{aligned}
$$

According to the Mercer Theorem (Steinwart and Scovel, 2012), the derivatives of the kernel are equal to the derivatives of the feature map. Therefore, the derivatives of the kernel can be obtained in the form of:

$$
\begin{aligned}
K_{1}\left(t_{i}, t\right) & =\frac{\partial K\left(t_{i}, t\right)}{\partial t}=\varphi\left(t_{i}\right)^{T} \dot{\varphi}(t) \\
\frac{d}{d t} \hat{x}_{k}(t) & =\sum_{i=1}^{n} \alpha_{i}^{k} K_{1}\left(t_{i}, t\right)
\end{aligned}
$$

Step 4 Identification of unknown parameters, and model's prediction

$\hat{x}_{k}(t)$ and $\frac{d}{d t} \hat{x}_{k}(t)$ in Eq. (11) and Eq. (14) are the approximated values of the $k$-th state variable and its derivative. All the state variables $\hat{X}$ and their derivatives $\frac{d}{d t} \hat{X}$ can be obtained by using the above same procedure based on NLS-SVM (RBF kernel). After substituting $\hat{X}$ and $\frac{d}{d t} \hat{X}$ in Eq. (4), the linear or nonlinear algebraic equation with unknown parameters is constructed.

Finally, the unknown parameters can be obtained by solving the optimization problem as:

$$
\min _{\theta} \frac{1}{2} \sum_{i}^{n}\left\|e_{i}\right\|_{2}^{2}
$$

Subject to

$$
e_{i}=\frac{d}{d t} \hat{X}\left(t_{i}\right)-g\left(\hat{X}\left(t_{i}\right), T, \theta\right), i=1,2, \cdots n
$$

\section{FREE DECAY TESTS OF ROLL MODEL}

\subsection{TOWING TANK}

Free decay tests were carried out at the Towing Tank for Manoeuvres in Confined Water (co-operation Flanders Hydraulics Research and Ghent University) in Antwerp, Belgium (Delefortrie et al., 2016) . The main dimensions of the towing tank are displayed in Table 1. 
Table 1. The main dimensions of the Towing Tank for Manoeuvres in Confined Water.

\begin{tabular}{lcc}
\hline Item & Value & Units \\
\hline Total length & 87.5 & $\mathrm{~m}$ \\
Useful length & 68.0 & $\mathrm{~m}$ \\
Width & 7.0 & $\mathrm{~m}$ \\
Maximum water depth & 0.50 & $\mathrm{~m}$ \\
Length of the ship models & $3.5-4.5$ & $\mathrm{~m}$ \\
\hline
\end{tabular}

\subsection{SHIP MODEL}

A 1/90 scale model of Ultra Large Container Vessel (ULCV) was chosen to carry out free roll decay tests (Tello Ruiz, 2018). The main parameters and cross sectional view of the ship model are shown in the Table 2 and Figure 1.

Table 2. ULCV ship model parameters.

\begin{tabular}{ccccc}
\hline \multirow{2}{*}{ Item } & \multicolumn{2}{c}{ Model scale $(1 / 90)$} & \multicolumn{2}{c}{ Full scale } \\
\cline { 2 - 5 } & Value & Units & Value & Units \\
\hline$L_{O A}$ & 4.418 & $\mathrm{~m}$ & 397.6 & $\mathrm{~m}$ \\
$L_{P P}$ & 4.191 & $\mathrm{~m}$ & 377.2 & $\mathrm{~m}$ \\
$B$ & 0.627 & $\mathrm{~m}$ & 56.4 & $\mathrm{~m}$ \\
$D$ & 0.330 & $\mathrm{~m}$ & 29.7 & $\mathrm{~m}$ \\
$T_{M}$ & 0.145 & $\mathrm{~m}$ & 13.1 & $\mathrm{~m}$ \\
$m$ & 226.4 & $\mathrm{~kg}$ & 165046 & ton \\
$C_{b}$ & 0.6 & -- & 0.6 & -- \\
\hline
\end{tabular}

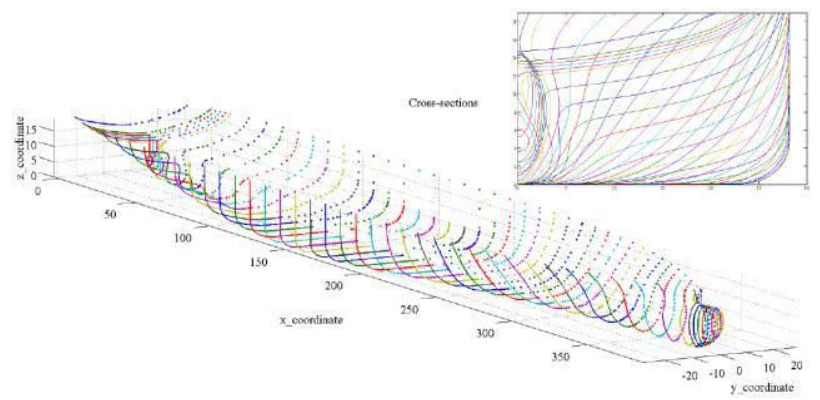

Figure 1. The cross sectional view of the ULCV ship.

\subsection{DECAY TESTS}

The free decay tests were performed by providing an initial roll angle of the ship model. Then, the ship model was hold at this initial position until the towing carriage reached its desired speed and immediately released by pulling the cord attached to a wooden stick (Tello Ruiz, 2018; Jang et al., 2010; Avalos et al., 2014). A illustration of the model test setup and mechanism is displayed in Figure 2.

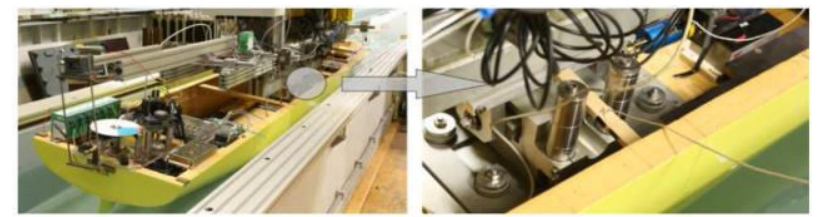

Figure 2. The model test setup and mechanism.
The free decay tests were carried out at different UKCs (from $20 \%$ to $190 \% \mathrm{UKC}$ ) and speeds (from $0 \mathrm{kn}$ to $21 \mathrm{kn}$ ). For the present study, the speeds at $0,6,12$ knots and the UKCs of $20 \%, 35 \%, 190 \%$ are selected as study cases. The initial roll angles at the chosen conditions are presented in Table 3.

Table 3. The initial roll angles at different speeds and UKCs.

\begin{tabular}{crcc}
\hline \multirow{2}{*}{ UKCs } & \multicolumn{3}{c}{ Speeds } \\
\cline { 2 - 4 } & $0 \mathrm{kn}$ & $6 \mathrm{kn}$ & $12 \mathrm{kn}$ \\
\hline $20 \% \mathrm{UKC}$ & $2.70^{\circ}$ & $2.71^{\circ}$ & $2.74^{\circ}$ \\
$35 \% \mathrm{UKC}$ & $3.11^{\circ}$ & $3.27^{\circ}$ & $1.95^{\circ}$ \\
$190 \% \mathrm{UKC}$ & $6.96^{\circ}$ & $6.45^{\circ}$ & $6.23^{\circ}$ \\
\hline
\end{tabular}

\section{PARAMETER IDENTIFICAION}

Taking into consideration of the parameters estimation method in the section 3, the novel NLS-SVM approach is introduced to estimate the linear and nonlinear viscous damping parameters in the nonlinear roll model. The identification process is, in more detail, described in Table 4 and Figure 3.

Table 4. Identification process of the NLS-SVM model. Parameter identification using NLS-SVM

1. Obtain the roll training sample $\left\{\left(t_{i}, \phi_{i}\right), i=\right.$ $1,2, \cdots, n\}$ based on numerical simulation tests or decay tests. $t_{i}$ is time series and $\phi_{i}$ is roll angles.

2. Estimate the trajectory of the roll angle $\phi$ (the state variable) by using NLS-SVM model, Eq. (11).

3. Differentiate the NLS-SVM predicting model with respect to time, Eq. (14); And the closed-form approximation for the first $(\dot{\phi})$ and second $(\ddot{\phi})$ derivatives of the state variable are obtained respectively.

4. Identify the linear $\left(b_{\dot{\phi}}\right)$ and nonlinear $\left(b_{\dot{\phi}|\dot{\phi}|}\right)$ viscous damping coefficients by solving the optimization problem in Eq. (15).

5. Substitute the identified linear and nonlinear viscous damping coefficients in the roll model, Eq. (3). After applying a fourth-order Runge-Kutta approach to solve the ship's roll response equation, the roll motions of the ship are predicted.

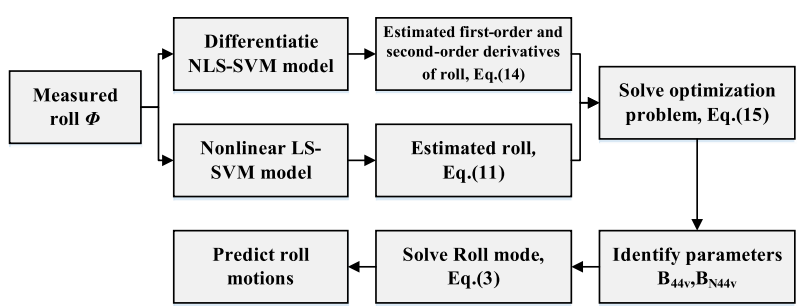

Figure 3. Identification process of NLS-SVM.

In order to verify the effectiveness, accuracy as well as applicability of the NLS-SVM model in shallow water, 
three case studies are conducted by using the novel identification method, they are:

Case 1. Validate the effectiveness of the NLS-SVM (5.1)

Case 2. Illustrate the advantages of the NLS-SVM (5.2)

Case 3. Verify the applicability of the NLS-SVM in shallow water (5.3)

These cases are described in detail in the following subsections.

\subsection{EFFECTIVENESS OF NLS-SVM MODEL}

In this case study, the roll motions with pre-defined linear $\left(b_{\dot{\phi}}\right)$ and nonlinear $\left(b_{\dot{\phi}}|\dot{\phi}|\right)$ viscous damping coefficients (Table 5) are selected to simulate a free roll decay test. The pre-defined linear and nonlinear damping parameters are substituted into the roll model, Eq. (3). It is worth noting that other parameters in Eq. (3) are regarded as known values, which can be found in Tello Ruiz (2018). Using a fourth-order Runge-Kutta approach to solve the differential equation the simulated roll angles (samples data) are obtained.

Subsequently, simulated samples data are divided into two sets, the first set as a training sample (blue circles) is used to train the NLS-SVM model and the second set as a test sample (green circles) is selected to test the model. The training results are displayed in Figure 4 (a).

After the NLS-SVM model being trained, unknown damping coefficients are identified by the proposed model and are shown in Table 5. Comparing the identified parameters with the pre-defined parameters, the results show that the relative errors of the linear $\left(b_{\dot{\phi}}\right)$ and nonlinear $\left(b_{\dot{\phi}|\dot{\phi}|}\right)$ viscous damping coefficients are about $0.3 \%$ and $1.0 \%$, respectively. These very small errors reveal that the NLS-SVM approach can accurately identify unknown parameters and can be well applied in identifying the roll model.

Furthermore, for a better idea of the importance of the method, the identified damping parameters are used to predict the roll motions and are compared against sample data. Satisfactory agreement between predicted values and original values can be found in Figure 4(b), with maximum errors between predicted values and original values of around 0.001 . This illustrates the potential of the NLS-SVM as it performs well with high prediction accuracy. Therefore, the novel NLS-SVM algorithm can be applied in identifying the ship roll model.

Table 5. Pre-defined parameters and identified parameters using the NLS-SVM approach.

\begin{tabular}{llll}
\hline Parameters & Known & Identified & Error $(\%)$ \\
\hline$b_{\dot{\phi}}\left(10^{8}\right)$ & 6.0 & 6.0171 & 0.2850 \\
$b_{\dot{\phi}|\dot{\phi}|}\left(10^{7}\right)$ & 4.0 & 4.0417 & 1.0425 \\
\hline
\end{tabular}

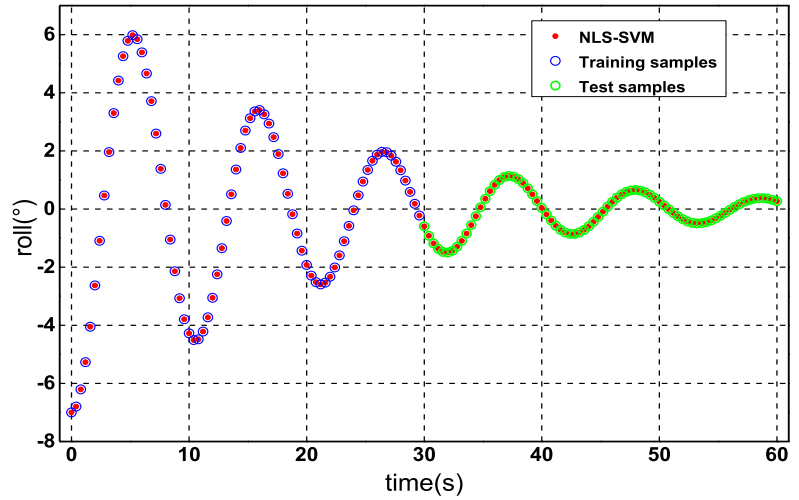

(a) Training results
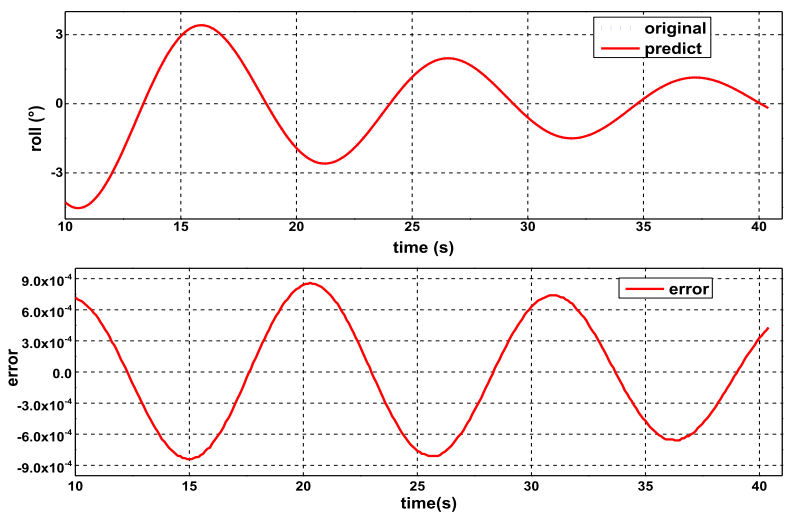

(b) Predicted results and errors

Figure 4. Training and predicted results with predefined damping coefficients.

\subsection{COMPARISON WITH DIFFERENT IDENTIFICATION METHODS}

In order to illustrate the advantages of the NLS-SVM algorithm, traditional methods, such as Nonlinear Least Square (NLS) and Fitting Least Square (FLS) algorithms are selected for comparing and analyzing.

The NLS algorithm has been one of the most common approaches used to identify unknown parameters before intelligent algorithms appeared (Zhu et al., 2017a). The method is described in Figure 5.

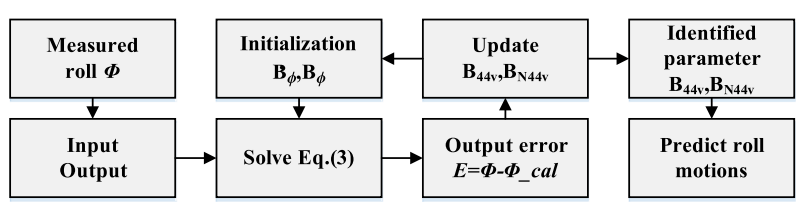

Figure 5. Nonlinear Least Square (NLS) identification method.

Apart from the NLS, the FLS algorithm has also been successfully applied for system identification. The main difference is that in the FLS approach the curve fitting is firstly applied to the initial data, then a basic LS algorithm is applied to identify the unknown parameters. This process is relatively simpler than the direct application 
displayed Figure 5, Figure 6 sketches the process for better illustration purposes.

Measured
roll $\Phi$$\rightarrow$ Curve Fitting $\rightarrow$ Solve Eq.(3) $\rightarrow \begin{gathered}\text { Identified } \\ \text { parameters } \\ \mathbf{B}_{44 \mathrm{v}}, \mathbf{B}_{\mathrm{N} 44 \mathrm{v}}\end{gathered} \rightarrow \begin{gathered}\text { Predict roll } \\ \text { motions }\end{gathered}$

Figure 6. Fitting Least Square (FLS) identification method.

Aiming at comparing and analyzing the performance of the three identified approaches, three evaluation indexes are selected, they are the Mean Absolute Error (MAE), the Root Mean Square Error (RMSE) (Zhu et al., 2017b), and the Computational time (CPU time) (Huang et al., 2018). In machine learning theory, the MAE is employed to assess the performance of the model; the RMSE is utilized to measure the accuracy of the model; the CPU time is represented as time and energy cost (Zhang et al., 2018). The MAE and the RMSE are, respectively given by:

$$
\begin{aligned}
\text { MAE } & =\frac{\sum_{n}^{i=1}\left|\phi_{i}-\widehat{\phi}_{i}\right|}{n} \\
\text { RMSE } & =\sqrt{\frac{\sum_{n}^{i=1}\left(\phi_{i}-\widehat{\phi}_{i}\right)^{2}}{n}}
\end{aligned}
$$

In this case study, the decay tests data at the speed of 12 knot and the UKC of $20 \%$ are selected to compare and analyze. The parameters identified by the methods described above and their respective comparison can be found in Table 6. From the results, it can be seen that the linear damping coefficient $\left(b_{\dot{\phi}}\right)$ can be identified by all methods within the same magnitude $\left(10^{8}\right)$. The nonlinear term, however, has some discrepancies, the traditional NLS method estimates a magnitude of $b_{\dot{\phi}|\dot{\phi}|}$ which is around 100 times smaller than other two approaches. The latter, draws the main attention to consider the use of intelligent method, the NLS-SVM, for improving the performance in practice.

Moreover, the MAE is around 0.0405 for the NLS-SVM model, which decreased by $27.9 \%$ from 0.0562 for the NLS model and by $17.2 \%$ from 0.0489 for the FLS model respectively. For accuracy analysis, the RMSE (0.0502) of the NLSSVM is reduced by $20.6 \%$ (0.0632) compared to the NLS model and by $17.4 \%(0.0608)$ compared to the FLS model, which demonstrates that the NLS-SVM model's errors are smaller, and the accuracy is higher than traditional methods. Moreover, comparing the CPU times, less time and energy are taken by the proposed model.

$$
\text { error }=\phi_{\text {known }} \phi_{\text {pred }}
$$

Table 6. Comparisons among different methods.

\begin{tabular}{lccc}
\hline \multirow{2}{*}{ Parameters } & \multicolumn{3}{c}{ Methods } \\
\cline { 2 - 4 } & NLS & FLS & NLSSVM \\
\hline$b_{\dot{\phi}}\left(10^{8}\right)$ & 3.30 & 3.43 & 3.38 \\
$b_{\dot{\phi}|\dot{\phi}|}\left(10^{7}\right)$ & 0.0493 & 5.14 & 3.55 \\
MAE & 0.0562 & 0.0489 & 0.0405 \\
RMSE & 0.0632 & 0.0608 & 0.0502 \\
CPU time(s) & 97 & 13 & 12 \\
\hline
\end{tabular}

Subsequently, the identified linear and nonlinear damping coefficients are employed to predict the ship's roll motions separately. The predicted results and errors (Eq. (19)) are presented in Figure 7(a) and 7(b), respectively. From Figure 7(a), there are small but not significant deviations between the original data and predicted data for the three identification approaches. It is noted that the predicted values by the NLS-SVM method are closer to the original values than the other two approaches. Furthermore, the overall errors of the NLS-SVM model in Figure 7(b) are smaller than that of NLS and FLS methods. In conclusion, the analyzed results demonstrate that the new NLS-SVM model has better identification performance and time saving ability as well as higher accuracy compared to traditional algorithms. The advantages of the NLS-SVM are validated.

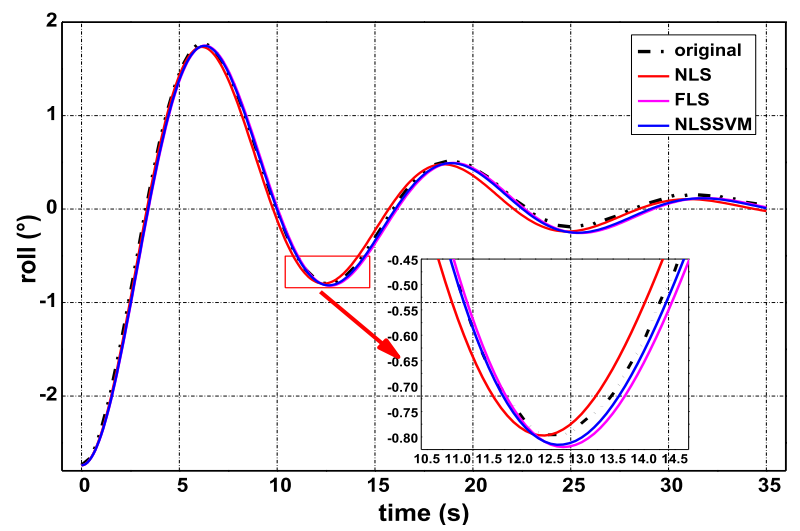

(a) Predicted results

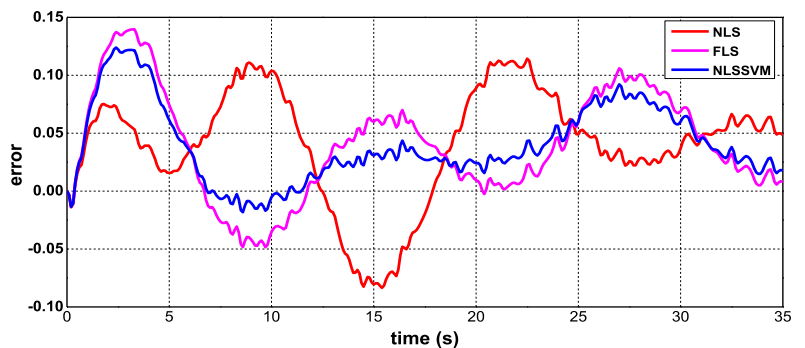

(b) errors

Figure 7. Predicted results and errors among different methods.

\subsection{APPLICATIONS ANALYSIS OF NLS-SVM}

In this section, the free roll decay tests from various UKCs and speeds are studied. The applicability in shallow water of the NLS-SVM model is illustrated.

\subsubsection{Applicability analysis for different UKCs}

Considering the effect of water depth on the damping coefficients, three different UKCs $(20 \%, 35 \%$ and $190 \%$ UKC) at a speed of 6 knots are selected as case studies. After the NLS-SVM model being trained by the decay 
tests data in different $\mathrm{UKCs}$, Table 7 presents a quantitative comparison of the identified results. It is noted that the RMSE (the values around 0.022-0.072) and the MAE (around 0.018-0.051) are pretty low, and computational time is very short (10-20s), which verify good generality and applicability of the NLS-SVM model both in shallow water and deep water.

Table 7. Comparisons among different UKC.

\begin{tabular}{lccc}
\hline \multirow{2}{*}{ Parameters } & \multicolumn{3}{c}{ UKC (6 knot) } \\
\cline { 2 - 4 } & $20 \%$ UKC & $35 \%$ UKC & $190 \%$ UKC \\
\hline$b_{\dot{\phi}}\left(10^{8}\right)$ & 1.97 & 1.70 & 0.95 \\
$b_{\dot{\phi}|\dot{\phi}|}\left(10^{7}\right)$ & 3.01 & 3.26 & 4.03 \\
MAE & 0.0177 & 0.0124 & 0.0510 \\
RMSE & 0.0223 & 0.0143 & 0.0729 \\
CPU time & 15 & 11 & 18 \\
\hline
\end{tabular}

Substituing the identified parameters in the roll model Eq. (3), ship roll motions are predicted for different UKCs. In Figure 8(a), 8(b) and 8(c), the predicted roll angles agree well with the original values from decay tests for different UKCs. In addition, the maximum errors of different UKCs are about $0.06,0.02$ and 0.2 , which are obtained at lower roll angles, and the errors could be a source of the model formulation problem and not of the identification method. The results show that the NLS-SVM method can successfully be used to identify parameters of the roll model in the shallow and deep water with small errors.
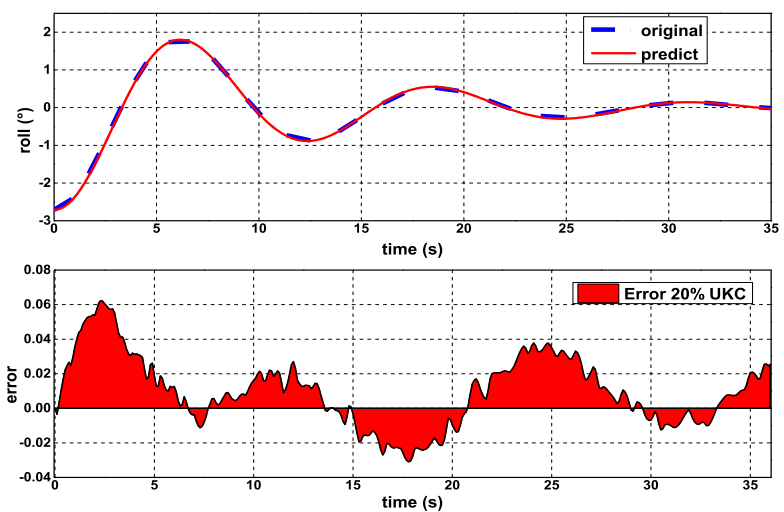

(a) Predicted results and errors for $20 \%$ UKC
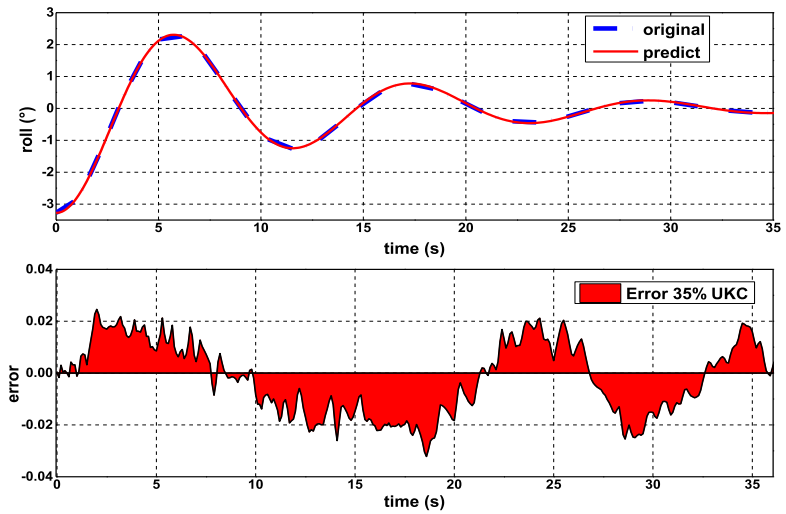

(b) Predicted results and errors for $35 \% \mathrm{UKC}$
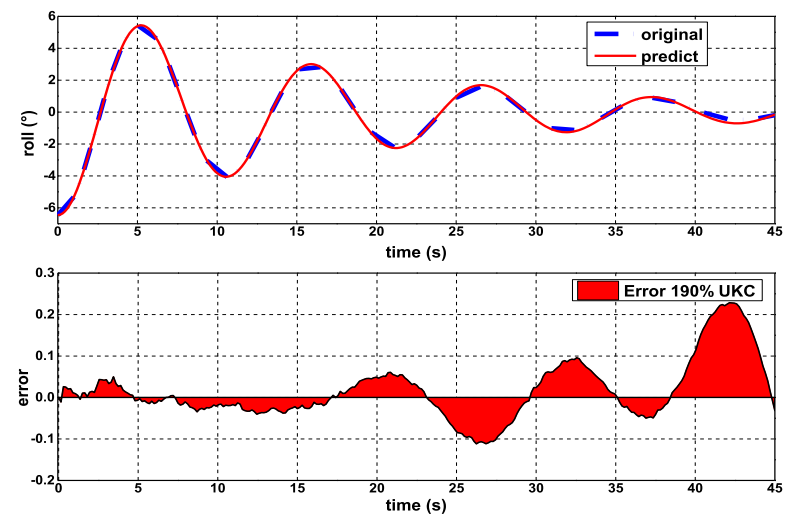

(c) Predicted results and errors for $190 \%$ UKC

Figure 8. Predicted results and errors in different UKCs.

\subsubsection{Applicability analysis for different speeds}

Similarly, the applicability in shallow water $(20 \%$ UKC) at different speeds is investigated. The experimental data at $0,6,12$ knots are taken as examples to train the NLSSVM model and unknown linear and nonlinear damping coefficients at different speeds are estimated. The identified results are shown in Table 8 . It can be seen that the three evolution indexes are very small. The RMSE is around $0.0185,0.0223$ and 0.0502 respectively, whose value increase slightly with higher speed. Moreover, the MAE at different speeds is with small values about 0.0185 to 0.0452 . For the CPU time, it is equal (15s). After quantitative comparison, it can be concluded that the NLSSVM model has good applicability in shallow water at different speeds.

Table 8. Comparisons among different speeds.

\begin{tabular}{llll}
\hline \multirow{2}{*}{ Parameters } & \multicolumn{3}{c}{ Speed (20\% UKC) } \\
\cline { 2 - 4 } & $0 \mathrm{kn}$ & $6 \mathrm{kn}$ & $12 \mathrm{kn}$ \\
\hline$b_{\dot{\phi}}\left(10^{8}\right)$ & 1.93 & 1.97 & 3.38 \\
$b_{\dot{\phi}|\dot{\phi}|}\left(10^{7}\right)$ & 2.66 & 3.01 & 3.55 \\
MAE & 0.0185 & 0.0177 & 0.0405 \\
RMSE & 0.0185 & 0.0223 & 0.0502 \\
CPU Time(s) & 15 & 15 & 15 \\
\hline
\end{tabular}

Furthermore, the predicted results in shallow water at different speeds are obtained in Figure 9(a), 9(b) and 9(c). It can be seen that the predicted values have satisfactory agreement with the original data at different speeds. The predicted roll angles at a speed of $12 \mathrm{kn}$ are a little bit higher than the original ones, but the predicted results are still valid, because the overall errors are very small and the effect on the ship is not significant.

To summarize, the effectiveness and applicabilty of the NLS-SVM approach applied in shallow water at different speeds are verified. 

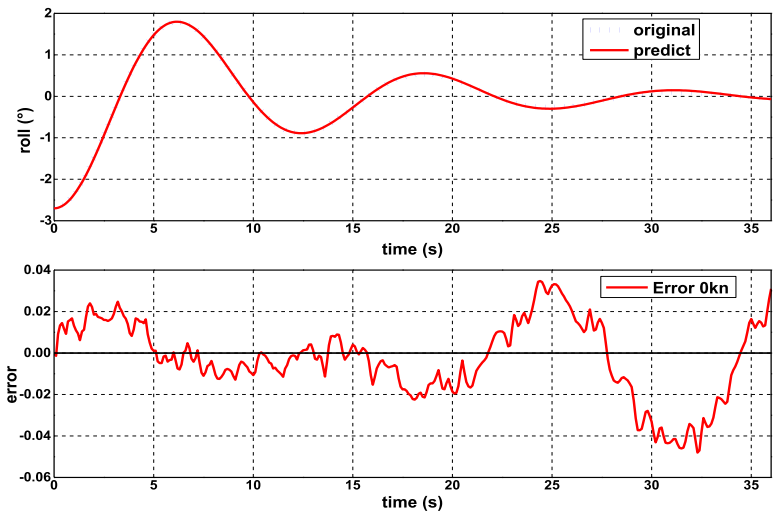

(a) Predicted results and errors at $0 \mathrm{kn}$
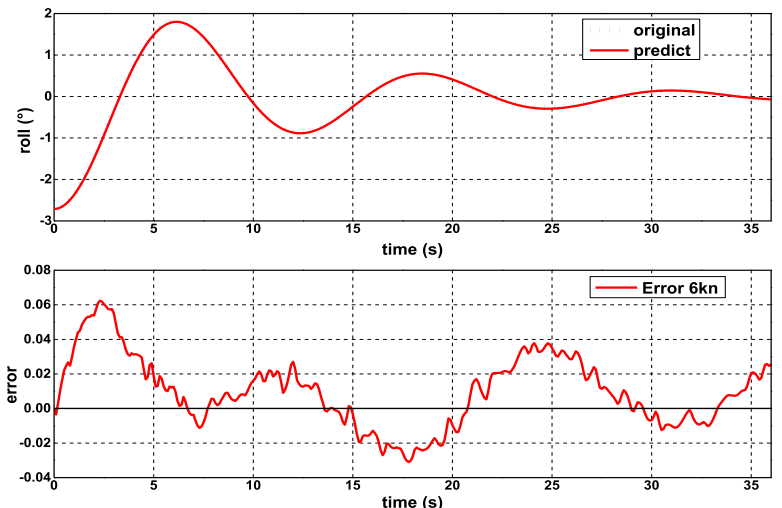

(b) Predicted results and errors at $6 \mathrm{kn}$
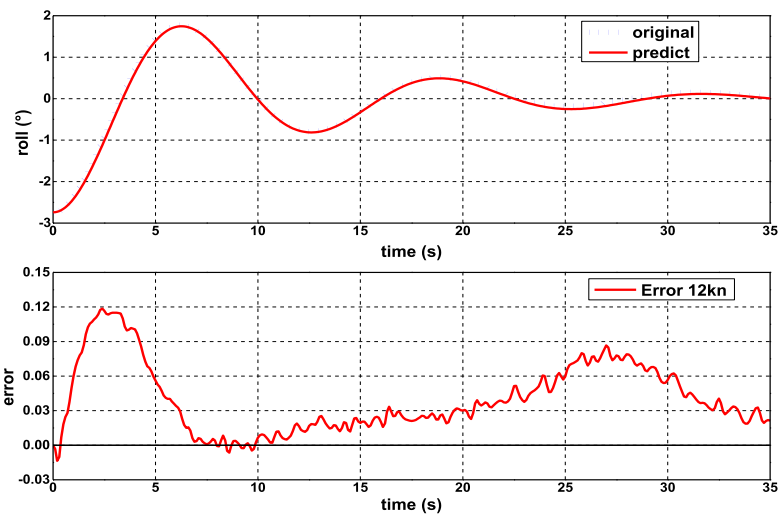

(c) Predicted results and errors at $12 \mathrm{kn}$

Figure 9. Predicted results and errors at different speeds.

\section{CONCLUSIONS}

In this paper, the novel NLS-SVM parametric identification approach for estimating unknown damping coefficients in shallow water is investigated. Firstly, comparisons between simulation roll angles based on predefined damping coefficients and predicted roll angles using estimated damping coefficients presents satisfactory agreement, which illustrate the proposed identification algorithm can be effectively applied in identifying the roll model. Subsequently, comparing traditional identification approaches (NLS and FLS) with intelligent method, the
NLS-SVM algorithm can perform better with higher accuracy, and overcome the weakness of conventional methods for identifying nonlinear damping coefficients. Moreover, decay tests data in different UKCs and speeds were prepared for the purpose of demonstrating the shallow water effect in parametric identification based on the NLS-SVM method. The good agreement between decay tests results and predicted results suggests the satisfactory applicability of the proposed algorithm in shallow water. Therefore, the effectiveness, accuracy and applicability of the NLS-SVM model applied in identifying the roll model in shallow water, have been verified by qualitative and quantitative analysis.

Future work includes two aspects: first, other parameters in roll model will be identified and analyzed. In addition, intelligent optimized algorithms will be considered as well as to obtain the best penalty factors and kernel factors of the NLS-SVM model.

\section{ACKNOWLEDGEMENTS}

This work was supported by the program of China Scholarships Council (201706570007, 201806230196) and Special Research Fund - Cofunding for Chinese candidates holding a CSC-grant (01SC8418).

\section{REFERENCES}

Avalos, G.O.G., Wanderley, J.B.V., Fernandes, A.C., Oliveira, A.C., 2014. Roll damping decay of a FPSO with bilge keel. Ocean Eng. 87, 111-120.

David, M.S., Dictino, C., Jesus Manuel, D.L.C., Joaquin, A., 2013. Identification of a surface marine vessel using LS-SVM. J. Appl. Math. 2013, 1-11.

Delefortrie, G., Geerts, S., Vantorre, M., 2016. The towing tank for manoeuvres in shallow water. In: Proceedings of 4th MASHCON. Hamburg, Germany, pp. 226-235.

Himeno, Y., 1981. Prediction of ship roll damping - state of the art. Technical Report of Department of Naval Architecture and Marine Engineering. University of Michigan, Michigan, USA.

Hou, X.R., Zou, Z.J., 2015. SVR-based identification of nonlinear roll motion equation for FPSOs in regular waves. Ocean Eng. 109, 531-538.

Hou, X.R., Zou, Z.J., 2016. Parameter identification of nonlinear roll motion equation for floating structures in irregular waves. Appl. Ocean Res. 55, 66-75.

Hou, X.R., Zou, Z.J., Liu, C., 2018. Nonparametric identification of nonlinear ship roll motion by using the motion response in irregular waves. Appl. Ocean Res. 73, 88-99.

Huang, B.G., Zou, Z.J., Ding, W.W., 2018. Online prediction of ship roll motion based on a coarse and fine tuning fixed grid wavelet network. Ocean Eng. 160, 425437.

Ikeda, Y., Himeno, Y., Tanaka, N., 1977. On eddy making component of roll damping force on naked hull. J. Soc. Nav. Archit. Japan. 1977(142), 54-64. 
Jang, T.S., Kwon, S.H., Lee, J.H., 2010. Recovering the functional form of the nonlinear roll damping of ships from a free-roll decay experiment: An inverse formulism. Ocean Eng. 37, 1337-1344.

Jiang, Y., Zhu, R., Miao, G., Yang, C., 2016. Damping prediction of a rolling ship with bilge keel in viscous flow based on numerical simulation. Shipbuild. China. 57(2), $1-12$.

Jiang, Y., Zhu, R.C., Fan, J., Ma, C., 2017. Nonparametric identification of nonlinear damping and restoring forces for ship free roll decay in numerical tank. J. Mar. Sci. Technol. 25(4), 404-416.

Mehrkanoon, S., Falck, T., Suykens, J.A.K., 2012. Parameter estimation for time varying dynamical systems using least squares support vector machines. IFAC Proc. Vol. 45(16), 1300-1305.

Steinwart, I., Scovel, C., 2012. Mercer's theorem on general domains: on the interaction between measures, kernels, and RKHSs. Constr. Approx. 35(3), 363-417.

Sun, L.P., Sun, W. Ben, 2013. Parameter identification of the non-linear rolling damping based on PLS regression technique. Adv. Mater. Res. 779(1), 675-679.

Tello Ruiz, M., 2018. Manoeuvring model of a container vessel in coastal waves. Ghent University, Belgium.

Xing, Z., McCue, L., 2010. Modeling ship equations of roll motion using neural networks. Nav. Eng. J. 122(3), 49-60.

$\mathrm{Xu}$, H.T., Guedes Soares, C., 2016. Vector field path following for surface marine vessel and parameter identification based on LS-SVM. Ocean Eng. 113, 151161.

Yin, J.C., Perakis, A.N., Wang, N., 2018. A real-time ship roll motion prediction using wavelet transform and variable RBF network. Ocean Eng. 160, 10-19.

Zhang, X.K., Zhang, Q., Ren, H.X., Yang, G.P., 2018. Linear reduction of backstepping algorithm based on nonlinear decoration for ship course-keeping control system. Ocean Eng. 147, 1-8.

Zhu, M., Hahn, A., Wen, Y., Bolles, A., 2017 a. Comparison and optimization of the parameter identification technique for estimating ship response models. In: 2017 IEEE 3rd International Conference on Control Science and Systems Engineering. Beijing, China, pp. 743-750.

Zhu, M., Hahn, A., Wen, Y.Q., Bolles, A., 2017 b. Identification-based simplified model of large container ships using support vector machines and artificial bee colony algorithm. Appl. Ocean Res. 68, 249-261.

\section{AUTHORS BIOGRAPHY}

Changyuan Chen, a $\mathrm{PhD}$ candidate at the Maritime Technology Division, which belongs to the Faculty of Engineering and Architecture of Ghent University. His experience includes research on trajectory controller, intelligent control strategies, machine learning techniques applied to ship hydrodynamics, etc.

Manases Tello Ruiz PhD, Naval Architect and Marine Engineer, is a Research Staff at Ghent University. He has been involved in several (inter-)national projects with main focus on manoeuvring, seakeeping, and wave energy converters. Currently, he is working on ship air pollution and machine learning techniques applied to ship hydrodynamics. At present he is also a member of the ITTC Specialist Committee of Manoeuvring in Waves, at which he has been appointed as secretary.

Guillaume Delefortrie, $\mathrm{PhD}$, naval architect, is expert nautical researcher at Flanders Hydraulics Research and visiting professor at Ghent University. He is in charge of the research in the Towing Tank for Manoeuvres in Confined Water and the development of mathematical models based on model tests. He has been secretary of the $27_{\text {th }}$ and 28th ITTC Manoeuvring Committee and is chairman of the 29th ITTC Manoeuvring Committee.

Tianlong Mei, $\mathrm{PhD}$ student at School of Naval Architecture, Ocean and Civil Engineering, Shanghai Jiao Tong University and the division of Maritime Technology at Ghent University. His experience includes numerical studies on wave structure interaction and ship manoeuvring in waves.

Evert Lataire, $\mathrm{PhD}$, naval architect, is currently postdoctoral assistant at the division of Maritime Technology at Ghent University. He has written a PhD on the topic of bank effects mainly based upon model tests carried out in the shallow water towing tank of FHR. His fifteen year experience includes research on ship manoeuvring in shallow and confined water such as shipship interaction, ship-bottom interaction and ship-bank interaction.

Marc Vantorre, naval architect, is full senior professor of marine hydrodynamics and head of the Maritime Technology Division at Ghent University, Belgium. His research focuses on ship behaviour in shallow and confined waters, mainly in close co-operation with Flanders Hydraulics Re-search in Antwerp. He is former member of PIANC Working Groups and of the ITTC Manoeuvring Committee. 